\title{
A Study of Instrument Motion in Retinal Microsurgery
}

\author{
Cameron N. Riviere ${ }^{1}$, Patrick S. Jensen ${ }^{2}$
}

\begin{abstract}
This paper reports on high-precision recordings of hand-held instrument motion during actual vitreoretinal microsurgery. The movement of a hand-held instrument during vitreoretinal microsurgery was recorded in six degrees of freedom. Data were acquired for $5 \mathrm{~min}$ using an inertial sensing module that has been developed for use with a commercially available microsurgical instrument. Maximum velocity used by the surgeon was estimated at $0.70 \mathrm{~m} / \mathrm{s}$, and maximum acceleration at $30.1 \mathrm{~m} / \mathrm{s}^{2}$. The $\mathrm{rms}$ amplitude of tremor in the instrument tip motion was estimated to be $0.182 \mathrm{~mm}$.
\end{abstract}

Key words - Microsurgery, accuracy, tremor, sensors

\section{INTRODUCTION}

Manual accuracy in microsurgery has been a focus of attention for some time, but quantitative assessment of the problem has been lacking. The result is that efforts toward development of systems for enhancement of microsurgical accuracy [1-3] are going forward without a complete description of the problem that is to be solved. The scarcity of data is exacerbated by the fact that those studies that have been done, e.g., studies of physiological tremor and other undesired components of hand movement, have been conducted under laboratory conditions [4,5]. Application of the results of such studies therefore requires consideration of the degree of surgical realism in the study, and its effect on the relevance of the data. Quantification of instrument motion during actual microsurgery would obviate this consideration. The capability of directly recording instrument motion during vitreoretinal microsurgery would allow quantification of various types of undesired motion (e.g., tremor, jerk) in authentic conditions, as well as determination of the "performance envelope" utilized by the surgeon in terms of velocity, acceleration, and frequency. All of these measurements would provide valuable baseline data for future development of accuracy-enhancement systems.

This paper describes the first set of data recorded during retinal microsurgery using a new retinal microsurgical instrument with six-degree-of-freedom (6-dof) inertial motion sensing [6]. To our knowledge, these are the first highprecision measurements of instrument motion ever taken during vitreoretinal microsurgery with hand-held instruments.

\section{METHODS}

An instrument for intraoperative tremor monitoring in vitreoretinal microsurgery was constructed and tested in the laboratory by adding a 6-dof inertial sensor suite to a commercially available instrument, the DP9603 Madlab Pic-Manipulator (Storz Ophthalmics, Inc., St. Louis, Mo.) [6]. The DP9603 was chosen because it is used for delicate manipulation of the retina. The sensor suite is $2.8 \mathrm{~cm} \times 3.3 \mathrm{~cm} \mathrm{x} 4.1 \mathrm{~cm}$ and adds $38 \mathrm{~g}$ to the $11 \mathrm{~g}$ mass of the instrument itself. The sensing module incorporates a CXL02LF3 tri-axial low-noise accelerometer (Crossbow Technology, Inc., San Jose, Ca.), and three CG-16D ceramic rate gyros (Tokin Corp., Tokyo).

Data were recorded during an epiretinal membrane removal procedure at The Johns Hopkins Hospital. The procedure took place under normal surgical conditions, with the surgeon viewing the procedure through a stereo operating microscope. Readings from the six sensors were sampled at $100 \mathrm{~Hz}$ for $5 \mathrm{~min}$ while the instrument was being used to manipulate the retina and pathological membranes. Both surgeon and patient gave written consent to the experiment under a board-approved protocol.

Given the $100 \mathrm{~Hz}$ sampling rate, angles traversed in any single time step are small, and correct results can be obtained by treating the three orthogonal rotations as sequential rather than simultaneous. A $Z-Y-X$ Euler angle formulation was therefore used to represent rotations. Data were lowpass filtered at $20 \mathrm{~Hz}$ to minimize noise, and highpass filtered at 0.1 $\mathrm{Hz}$ to suppress drift, as well as the effect of gravity on the accelerometers. The gyro measuring rotation about $\mathrm{Z}$ (with $\mathrm{Y}$ being the lengthwise axis of the instrument) exhibited a large noise peak at roughly $5.5 \mathrm{~Hz}$, therefore notch filtering at this frequency was used on this single channel of data. Using appropriate kinematic calculations [7], the velocity of the instrument tip with respect to a fixed reference frame was calculated. Displacement and acceleration were then estimated by integration and differentiation. Tremor amplitude was estimated by bandpass filtering with cutoffs of 7 and $13 \mathrm{~Hz}$,

\footnotetext{
1 The Robotics Institute

Carnegie Mellon University

Pittsburgh, PA 15213 USA

cam.riviere@ri.cmu.edu

2 The Wilmer Eye Institute

The Johns Hopkins Hospital

Baltimore, MD 21287 USA

psjensen@jhu.edu
} 
and then computing the rms amplitude. A frequency spectrum was also estimated for the $X$ coordinate of tip displacement. Maximum velocity and acceleration utilized were taken from the absolute values of the velocity and acceleration vectors, and maximum frequency utilized was estimated from the spectral data.

\section{RESULTS}

Table 1 presents the maximum utilized velocity and acceleration as computed from the data. The rms amplitude of physiological tremor was $0.182 \mathrm{~mm}$. Figure 1 presents the power spectrum of the $X$ coordinate of the displacement data. The power at $3 \mathrm{~Hz}$ is roughly one-hundredth that at zero frequency, and that at $5 \mathrm{~Hz}$ is roughly one-thousandth that at zero frequency.

Table 1. Performance EnVelope Maximum values of relevant quantities measured from data

\begin{tabular}{ll}
\hline Velocity $(\mathrm{m} / \mathrm{s})$ & Acceleration $\left(\mathrm{m} / \mathrm{s}^{2}\right)$ \\
\hline 0.70 & 30.1 \\
\hline
\end{tabular}

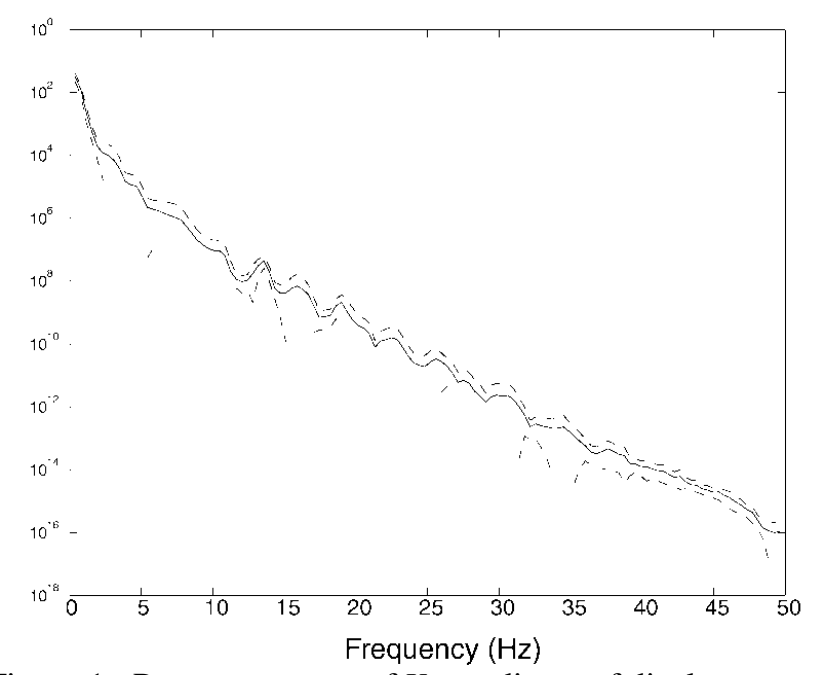

Figure 1. Power spectrum of $X$ coordinate of displacement data.

\section{DISCUSSION}

Integration of inertial sensor data to obtain displacement exhibits considerable drift over a five-minute period. However, the displacement estimate was used only to estimate tremor amplitude (after bandpass filtering), and to estimate the power spectrum in Fig. 1, therefore effects of this drift on the results were not significant.

It is expected that the weight added to the instrument by the sensor module attenuated the tremor somewhat. However, the DP9603 is a relatively lightweight instrument for this field. The range of commercially available instruments for vitreoretinal microsurgery includes instruments weighing more than the sensor-equipped instrument used here. In the future, a sensor module approaching one-tenth the weight of the present one is envisioned.

In addition to enabling basic studies of hand motion in microsurgery, the sensing scheme described herein is a fundamental step in the development of an intelligent handheld instrument for active tremor canceling in microsurgery [3].

\section{CONCLUSION}

Fundamental data regarding hand motion have been recorded during vitreoretinal microsurgery using a new retinal pick equipped with inertial motion sensing. Data obtained include information regarding tremor and other subcomponents of motion, as well as frequency band and envelopes of velocity and acceleration.

\section{ACKNOWLEDGMENTS}

Funding provided by Johnson \& Johnson Focused Giving Program and National Science Foundation (grant no. EEC9731748). Thanks to Dante Pieramici, M.D., for participation.

\section{REFERENCES}

[1] P. S. Schenker, E. C. Barlow, C. D. Boswell, H. Das, S. Lee, T. R. Ohm, E. D. Paljug, G. Rodriguez, and S. T. Charles, "Development of a telemanipulator for dexterity enhanced microsurgery," Proc. 2nd Intl. Symp. Med. Robot. Comput. Assist. Surg., pp. 81-88, 1995.

[2] R. Taylor, P. Jensen, L. Whitcomb, A. Barnes, R. Kumar, D. Stoianovici, P. Gupta, Z. Wang, E. deJuan, and L. Kavoussi, "A steady-hand robotic system for microsurgical augmentation,” in: C. Taylor, A. Colchester (eds.), Medical Image Computing and Computer-Assisted Intervention--MICCAI'99. Springer, Berlin, 1999, pp. 1031-1041.

[3] C. N. Riviere, R. S. Rader, and N. V. Thakor, "Adaptive canceling of physiological tremor for improved precision in microsurgery," IEEE Trans. Biomed. Eng., vol. 45, pp. 839-846, 1998.

[4] R. C. Harwell and R. L. Ferguson, "Physiologic tremor and microsurgery," Microsurgery, vol. 4, pp. 187-192, 1983.

[5] C. N. Riviere, R. S. Rader, and P. K. Khosla, "Characteristics of hand motion of eye surgeons," Proceedings of the 19th Annual Conference of the IEEE Engineering in Medicine and Biology Society, Chicago, 1997.

[6] M. Gomez-Blanco, C. N. Riviere, and P. K. Khosla, "Intraoperative tremor monitoring for vitreoretinal microsurgery," Proc. Medicine Meets Virtual Reality 8, 2000, to appear.

[7] M. Gomez-Blanco, C. Riviere, and P. Khosla, "Sensing hand tremor in a vitreoretinal microsurgical instrument," tech. report CMU-RI-TR-99-39, Robotics Institute, Carnegie Mellon University, December, 1999. 